\title{
Hematological laboratory parameters: may act as a surrogate of chest radiographs in Pediatric COVID- 19 Pneumonia.
}

Karuna M Das. ( $\square$ daskmoy@gmail.com )

United Arab Emirates University

Jamal Aldeen Alkoteesh

Tawam Hospital

Usama M AlBastaki

Rashid Hospital

Rajvir Singh

All India Institute of Medical Sciences

Abbey J Winant

Boston Children's Hospital

Taleb Almansoori

United Arab Emirates University

Klaus V Gorkom

United Arab Emirates University

Edward Y Lee

Boston Children's Hospital

\section{Research Article}

Keywords: Ionizing Radiation Exposure, Ground-glass Opacity, Peribronchial Thickening, Elevated LDH

Posted Date: May 17th, 2021

DOl: https://doi.org/10.21203/rs.3.rs-455518/v1

License: (1) (1) This work is licensed under a Creative Commons Attribution 4.0 International License.

Read Full License 


\section{Abstract}

Background: COVID-19 infection continues to be a serious health concern in pediatric patients, associated with substantial morbidity and mortality. An important clinical question is whether laboratory hematologic parameters may act as a surrogate for imaging findings in pediatric COVID-19 pneumonia, in order to reduce exposure to ionizing radiation. The aim is to investigate the relationship between radiographic findings and hematological laboratory parameters in pediatric patients with COVID-19 infection by directly comparing the findings from both studies.

Methods: This was an institutional review board-approved retrospective study of 187 consecutive pediatric patients with microbiologically confirmed COVID-19 pneumonia and available initial chest radiographs at the time of diagnosis, obtained between March 2020 and December 2020. Two groups of patients were created: 1) 1st group (without radiographic evidence of COVID-19 pneumonia) and 2) second group (with radiographic evidence of COVID-19 pneumonia). Two experienced radiologists independently reviewed the initial chest radiographs for abnormalities. Patients' medical records were reviewed for clinical presentation, hematological laboratory parameters, and patient outcomes. The relationship between initial chest radiographic findings and hematological laboratory parameters was evaluated between the two groups. Interobserver agreement was estimated with the Cohen $\mathrm{k}$ coefficient.

Results: The study population consisted of 187 chest radiographs from 187 individual pediatric patients (95 males and 92 females; mean age \pm SD, $10.1 \pm 6.0$ years; range, 9 months -18 years). Group 1 consisted of 103 chest radiographs (55.0\%) from 103 individual pediatric patients (54 males and 49 females; mean age \pm SD, $12.5 \pm 5.2$ years; range, 9 months -18 years). Group 2 consisted of 84 chest radiographs (45.0\%) from 84 individual pediatric patients ( 41 males and 43 females; mean age \pm SD, 7.0 \pm 5.6 years; range, 11 months -18 years). In group 2, observed chest radiographic abnormalities included ground-glass opacity (GGO) in 75 patients (89.2\%), GGO and consolidation in 6 (7.2\%), peribronchial thickening in 1 patient $(1.1 \%)$, consolidation in 1 patient (1.1\%), and peribronchial thickening, GGO and consolidation in one patient (1.1\%). In addition, group 2 patients (with radiographic abnormalities of COVID-19 pneumonia) had significantly elevated LDH $(p=0.001)$ compared to group 1 patients (without radiographic findings of COVID-19 pneumonia). All pediatric patients in both groups completely recovered from COVID-19 infection. Interobserver agreement for chest radiographic findings was nearly perfect between the two reviewers in both groups $(k=0.96, p=0.001)$.

Conclusion: The presence of radiographic abnormalities on initial chest radiographs correlates well with hematologic laboratory abnormalities, specifically elevated LDH, in pediatric patients with COVID-19 pneumonia. Therefore, it is reasonable to conclude that hematologic laboratory parameters may act as a surrogate for chest radiography in pediatric COVID-19 pneumonia and that chest radiography may not be clinically indicated in pediatric patients with COVID-19 infection with normal LDH level, resulting in decreased exposure to ionizing radiation in this vulnerable population.

\section{Background}


COVID-19 pneumonia continues to be a serious health concern in pediatric patients, associated with substantial morbidity and mortality [1 - 3]. Chest radiography and computed tomography are two types of imaging studies (CT), are frequently utilized in conjunction with quantitative reverse transcriptasepolymerase chain reaction (RT-PCR) test for initial diagnosis and evaluation of disease progression in pediatric COVID-19 pneumonia [4 - 7]. Unfortunately, these imaging studies are associated with ionizing radiation exposure, which may be particularly harmful in the vulnerable pediatric population [8-10]. As a result, a surrogate test that can be used in lieu of imaging studies for the diagnosis and evaluation of COVID-19 infection in children is critical.

COVID-19 is a systemic infection that, while still poorly understood, has been shown to have a significant pathologic effect on multiple organ systems throughout the body, resulting in hematological laboratory abnormalities. Several recent publications reported altered hematological laboratory parameters in pediatric and adult populations [11 - 13]. An important clinical question is whether laboratory hematologic parameters may act as a surrogate for imaging findings in pediatric COVID-19 pneumonia to reduce exposure to ionizing radiation associated with imaging studies in the vulnerable pediatric population.

Currently, no published scientific study has examined the relationship between chest radiographic findings and hematological laboratory parameters in pediatric patients with COVID-19 pneumonia. By directly comparing the results from both studies, the aim of this research is to investigate the relationship between radiographic findings and hematological laboratory parameters in pediatric patients with COVID19 infection.

\section{Methods}

\section{Institutional Review Board Approval}

The approval of the retrospective study was obtained from our institutional review board (Department of health, Abu Dhabi) to examine chest radiographs, hematological laboratory parameters, and patient outcomes. A waiver of informed consent was obtained from the same institutional review board (Department of health, Abu Dhabi). The protocol was performed in accordance with the relevant guidelines and regulations. The patient's confidentiality was protected by closely following the Health Insurance Portability and Accountability Act (HIPAA) guidelines.

\section{Patient Population}

All consecutive pediatric patients ( 18 years old) diagnosed with COVID-19 infection from March 2020 to December 2020 were identified using our radiology department information system. The selection of patients was based upon: 1) COVID-19 infection confirmed by RT-PCR test from respiratory secretions using a nasopharyngeal or oropharyngeal swab according to Centers for Disease Control and Prevention criteria. [14] and 2) availability of both chest radiography and hematological laboratory test results on the first day of hospital administration. The final study population included 187 chest radiographs and 
corresponding hematological laboratory test results from 187 consecutive pediatric patients with COVID19 pneumonia.

Of note, 56 (29.9\%) out of 187 chest radiographs included in our study were also used for the previously published study, only focusing on chest radiographic and chest computed tomography (CT) correlation for detecting abnormal thoracic findings in pediatric patients with COVID-19 pneumonia [6].

\section{Imaging Technique}

All chest radiographs were obtained as a digital radiograph in anteroposterior (AP) (26 (13.9\%) or posteroanterior (PA) (161 (86.1\%) projection based on our department's standard technique with chest radiography equipment. AP projection radiograph technique included high voltage, tube current, and exposure times at a 100-cm focus-film distance with mobile radiography equipment (Digital Radiographic Mobile X-ray System; Shimadzu). PA projection radiograph technique included high voltage, tube current, and exposure times at a $180-\mathrm{cm}$ focus-film distance with mobile radiography equipment (Digital Diagnost; Philips Healthcare).

\section{Imaging Study Review}

Two reviewers (K.M.D. and J.K.) with more than 20 years of experience in interpreting pediatric chest radiographs independently reviewed all chest radiographs. These two reviewers were blinded to all patient information, including clinical history, prior imaging studies, and the original reports of chest radiographs that were included in this study population. The third reviewer (E.Y.L.), with 20 years of experience in interpreting pediatric chest radiography, served as a tie-breaker for discrepancies between the two reviewers' initial interpretations without knowledge of the initial reviewers' findings and patients' clinical and imaging study information. A standard picture archiving and communication system (Cedara I-Read 5.2 P11; Cerner Image Devices) was used for reviewing all chest radiographs included in this study.

\section{Imaging Study Evaluation and Categorization}

The reviewers first reviewed the chest radiographs for abnormalities according to "Fleischer Society: glossary of terms for thoracic imaging" for the presence of abnormalities in 1) lung parenchyma and airway (ground-glass opacity (GGO), consolidation, peribronchial thickening); 2) pleural (pleural effusion and pneumothorax), and 3) mediastinum (lymphadenopathy) [15]. Subsequently, the chest radiographs were categorized into two groups: 1) group 1 (without radiographic evidence of COVID-pneumonia) and 2) group 2 (with radiographic evidence of COVID-19 pneumonia) based on the criteria of radiographic findings of COVID-19 pneumonia in children from international expert consensus statement on chest imaging in pediatric COVID-19 patient management [7]. Among the final 187 chest radiographs included in this study, group 1 consisted of 103 chest radiographs (55.5\%), and Group 2 consisted of 84 chest radiographs $(44.5 \%)$.

\section{Hematological Laboratory Parameter Evaluation}


The patients' medical records were carefully reviewed for the following hematological laboratory test results at presentation, including 1) lactate dehydrogenase (LDH);2) C-reactive protein; 3) ferritin; 4) aspartate aminotransferase (AST); 5) alanine aminotransferase (ALT); 6) platelet; 7) lymphocytes; and 8) white blood cell (WBC) levels.

\section{Evaluation of Patient Outcomes}

The patient's medical record was reviewed for the length of hospital stay and patient outcome.

\section{Statistical Analysis}

Descriptive statistics were obtained in the form of mean with standard deviation (SD) for interval variables. The minimum and maximum range were calculated forage with mean and standard deviation. Frequency distribution with the percentage was calculated for categorical variables. For normally distributed interval variables, Student t-tests were used to compare the two groups group 1 (without radiographic evidence for COVID-19 pneumonia) and group 2 (with radiographic evidence for COVID-19 pneumonia), and the Mann Whitney $U$ test was used for non-normally distributed variables. Inter-observer reliability is calculated in Cohen's kappa statistics $(k)$ to assess the degree of agreement between the two reviewers $[16,17]$. A statistically significant level was defined as a P-value of 0.05 (two-tailed). The statistical analysis was performed using the SPSS 22.0 statistical software [18].

\section{Results}

\section{Patient population}

The final study population consisted of 187 chest radiographs from 187 individual pediatric patients ( 95 males and 92 females; mean age \pm SD, $10.1 \pm 6.0$ years; range, 9 months -18 years). Group 1 consisted of 103 chest radiographs (55.0\%) from 103 individual pediatric patients (54 males and 49 females; mean age \pm SD, $12.5 \pm 5.2$ years; range, 9 months -18 years). Group 2 consisted of 84 chest radiographs (44.5\%) from 84 individual pediatric patients (41 males and 43 females; mean age \pm SD, $7.0 \pm 5.6$ years; range, 11 months -18 years).

Among 187 patients, 103 patients (55.0\%) presented with mild disease, $83(44.4 \%)$ presented with moderate disease, and one remaining patient $(0.6 \%)$ presented with the severe disease based on the previously published study disease classification $[3,11]$. The duration of symptoms was not statistically different between the two groups ( $1.5 \pm 1.2$ days in group 1 vs. $1.7 \pm 1.0$ days in group 2$)(P=0.5)$.

\section{Chest Radiographic Findings}

The chest radiographic findings of patients included in this study are summarized in Table 1. No chest radiographic abnormality was detected in group 1 patients ((Figure 1). The abnormalities observed on chest radiographs in group 2 included ground-glass opacity (GGO) in 75 patients (89.2\%), GGO and consolidation in 6 (7.2\%), peribronchial thickening in 1 patient (1.1\%), consolidation in 1 patient $(1.1 \%)$, 
and peribronchial thickening, GGO, and consolidation in one patient (1.1\%) (Figure 2). No pleural effusion, pneumothorax, or mediastinal lymphadenopathy was observed in any cases.

\section{Interobserver Agreement on Chest Radiographic Findings}

Among 187 chest radiographs reviewed between the two initial reviewers, there were discrepant interpretations in three cases, all related to the presence of GGO. The third reviewer, a tie-breaker, independently evaluated these three cases without the knowledge of the initial reviewers' interpretations or original report findings and determined that GGO was present in all three cases. The two reviewers had almost perfect agreement in detecting the presence or absence of radiographic abnormalities in both groups $(k=0.96, p=0.001)$.

\section{Hematological Laboratory Parameter Findings and Correlation with Chest Radiographic Findings}

The details of the patients' hematological laboratory test results at presentation in group 1 versus group 2 are listed in Table 2. Group 2 pediatric patients (with radiographic evidence for COVID-19 pneumonia) had significantly elevated LDH $(200.6 \pm 53.4$ vs. $258.9 \pm 80.8, P=0.001)$ with a moderate elevation of C-reactive protein $(2.8 \pm 5.0$ vs. $5.7 \pm 12.3, P=0.05)$, serum Ferritin $(67.3 \pm 93.8$ vs. $93.2 \pm 172.0, P=0.25)$ and AST ( $24.8 \pm 15.2$ vs. $27.8 \pm 12.0, P=0.22)$ in comparison to the group 1 pediatric patients. There was no statistically significant difference noted in the remaining hematological laboratory parameters.

\section{Patient Outcome}

The mean length of hospital stay was longer for group 2 patients $(2.8+/-3.4$ days) than group 1 patients $(1.2+/-1.0$ days). The difference in hospital stay length between the two groups was significant $(P=0.001)$. All pediatric patients in both groups completely recovered from COVID-19 infection. After the hospitalization, all the pediatric patients were subsequently sent to a quarantine facility for a total of 14 days, including days spent in the hospital.

\section{Discussion}

This study shows a statistically significant correlation between the presence of chest radiographic abnormalities and hematological laboratory parameters at presentation. Specifically, elevated serum LDH levels were associated with chest radiographic abnormalities in pediatric COVID-19 pneumonia. Chest radiographs are associated with potentially harmful ionizing radiation exposure and often result in findings that are non-specific for the diagnosis of COVID-19 pneumonia in children $[4-10,19]$. Consequently, a surrogate hematologic laboratory marker, which can provide similar diagnostic information without associated ionizing radiation exposure, would be ideal for evaluating pediatric COVID-19 patients to decrease exposure to ionizing radiation. Based on this study's results, we believe that hematological laboratory parameters, namely LDH level, may act as a surrogate for chest radiography in pediatric COVID-19 infection. 
Both the hematologic and imaging results of COVID-19 infection have been studied since the beginning of the COVID-19 pandemic, but mostly separately. For example, several previous publications are focusing on the imaging findings of COVID-19 pneumonia in children in radiology journals and hematological laboratory parameters in clinical journals independently $[4-7,11]$. However, to our knowledge, no previously published study directly investigates the correlation between chest radiographic abnormalities and hematologic laboratory abnormalities in pediatric patients with COVID-19 pneumonia. Interestingly, our study results show that serum inflammatory markers, such as LDH, are elevated above the normal range, even in group 1 (pediatric patients without radiological findings of COVID-19 pneumonia), which constituted $45 \%$ of our study population. Based on this study's results, we believe that hematologic abnormalities may be more reliable than radiologic abnormalities for detecting underlying systemic inflammation in pediatric patients with COVID-19.

Although it is understandable that hematological markers of inflammation, such as serum LDH, Creactive protein, and ferritin, would be elevated in patients with any underlying infection, including COVID19 infection, these inflammatory biomarkers are currently considered important for prognosis in COVID19 pneumonia, as elevated inflammatory markers have been associated with a higher risk of ARDS, ICU admission, and mortality in COVID-19 pneumonia [2, 12, 13 and 20]. Interestingly, a previously published study that focused only on the clinical features of COVID-19 infection in children, consisting of 36 pediatric patients, also found altered hematological laboratory parameters, such as decreased lymphocytes [11]. However, their study did not specifically evaluate LDH level. A direct correlation between their study results and our study cannot be made because their study did not correlate hematologic findings with radiographic abnormalities, which was our current study's focus.

During the initial phase of COVID-19 infection, when the new disease was localized within the Hunan province in China, chest radiography and chest computed tomography (CT) were widely used as initial screening tools and for evaluation of disease severity in most COVID-19 patients, including children [21]. The American College of Radiology (ACR), the Fleischner Society, and an international expert consensus statement on pediatric COVID-19 patient management have recommended that imaging is not recommended for pediatric patients who test positive for COVID-19 by RT-PCR but are asymptomatic or have mild symptoms, and that chest CT should be reserved for patients with progressive disease $[7,22$, 23]. Furthermore, a recent study focusing on the added diagnostic value of chest CT versus chest radiography in pediatric patients with COVID-19 pneumonia supports the notion that CT is not indicated for the initial evaluation of mild to moderately symptomatic pediatric patients with COVID-19 pneumonia [6]. Our study results further contribute to the evidence that imaging studies with ionizing radiation, including chest radiographs and CT, may not be needed for the initial evaluation of COVID-19 in children if there is no hematological laboratory parameter abnormality. In other words, if hematological laboratory parameters are within normal limits in pediatric patients diagnosed with COVID-19 infection based on RTPCR test, those pediatric patients will likely not require imaging assessment (since chest radiography will most likely be normal in these patients). 
There are two main limitations in our study. First, the study population is relatively small. We would like to emphasize, however, that our retrospective study was comprised of a unique group of pediatric patients who had both chest radiographs and hematological laboratory test results obtained on the same day during initial admission to the hospital. Future studies with a larger pediatric patient population will help confirm the results of our study. Second, our study population had only a single frontal chest radiographic view without a lateral chest radiographic view, which may have limited evaluation, especially the ability to detect small pleural effusion or mediastinal lymphadenopathy. However, pleural effusion and/or mediastinal lymphadenopathy is rarely seen in pediatric patients with acute COVID-19 pneumonia based on previously published studies [4 - 7]. Therefore, we believe that the inclusion of only frontal chest radiographs most likely did not substantially affect our study results. We would also like to emphasize that performance of only single frontal chest radiographs was often done to decrease the ionizing radiation exposure.

In conclusion, the previously published investigations of pediatric COVID-19 focused on either clinical laboratory abnormalities or imaging abnormalities separately, whereas our study investigated the relationship between hematological laboratory parameters and chest radiographic findings. Our study results show a new and important finding: the presence of radiographic abnormalities on initial chest radiography correlates well with hematological laboratory abnormalities, specifically, elevated serum LDH level. Therefore, hematological laboratory parameters may be used as a surrogate for chest radiography in pediatric patients with COVID-19 pneumonia. This approach has a great potential to decrease chest radiography and, consequently, ionizing radiation exposure in pediatric patients with positive RT-PCR testing for COVID-19 infection.

\section{Declarations}

- Ethics approval and consent to participate: The approval of the retrospective study was obtained from our institutional review board (Department of Health, Abu Dhabi) to examine chest radiographs, hematological laboratory parameters, and patient outcomes. A waiver of informed consent was obtained from the same institutional review board (Department of Health, Abu Dhabi). The protocol was performed in accordance with the relevant guidelines and regulations. The patient's confidentiality was protected by closely following the Health Insurance Portability and Accountability Act (HIPAA) guidelines

- Consent for publication: Not applicable.

- Availability of data and materials: The datasets used and/or analyzed during the current study available from the corresponding author on reasonable request.Competing interests.

The authors declare that the research was conducted in the absence of any commercial or financial relationships that could be construed as a potential conflict of interest.

- Funding: Not taken.

- Authors' contributions: 
Drs. KMD and EYL, JK conceptualized and initiated the study, collected, analyzed, and interpreted the data, wrote and reviewed the manuscript.

Drs. EYL, conceptualized and initiated the study, collected and interpreted data, wrote and reviewed the manuscript.

Dr. AJW, conceptualized and initiated the study, interpreted data, wrote and reviewed the manuscript.

Drs. SB, KM, TM, KG provided the patient's clinical information and reviewed the manuscript. RS performed the statistical analysis. All authors approved the final manuscript as submitted and agree to be accountable for all aspects of the work.

- Acknowledgments: NA.

\section{Abbreviations}

$\mathrm{LDH}$, lactate dehydrogenase; $A S T$, aspartate aminotransferase; $\mathrm{ALT}$, alanine aminotransferase

\section{References}

1. Buonsenso D, Sali M, Pata D, De Rose C, Sanguinetti M, Valentini P, et al. Children and COVID-19: Microbiological and immunological insights. Pediatr Pulmonol. 2020;55(10):2547-2555.

2. Koh HK, Geller AC, VanderWeele TJ. Deaths from COVID-19. JAMA. 2021;325(2):133-134.

3. de Souza TH, Nadal JA, Nogueira RJN, Pereira RM, Brandão MB. Clinical manifestations of children with COVID-19: a systematic review. Pediatr Pulmonol. 2020;55(8):1892-1899.

4. Foust AM, Winant AJ, Chu WC, Das KM, Phillips GS, Lee EY. Pediatric SARS, H1N1, MERS, EVALI, and now coronavirus disease (COVID-19) pneumonia: what radiologists need to know. AJR Am J Roentgenol. 2020; 215(3): 736 - 744.

5. Foust AM, McAdam AJ, Chu WC, Garcia-Pena P, Phillips GS, Plut D, Lee EY. Practical guide for pediatric pulmonologists on imaging management of pediatric patients with COVID-19. Pediatr Pulmonol. 2020; 55(9): 2213 - 2224.

6. Das KM, Alkoteesh AJ, Al Kaabi J, et al. Comparison of chest radiography and chest CT for evaluation of pediatric COVID-19 pneumonia: Does CT add diagnostic value? Pediatr Pulmonol. 2021 Feb 25. doi: 10.10002/ppul.25313. Online ahead of print.

7. Foust AM, Phillips GS, Chu WC, et al. International expert consensus statement on chest imaging in pediatric COVID-19 patient management: imaging findings, imaging study reporting and imaging study recommendations. Radiol Cardiothorac Imaging. 2020; 23; 2(2): e200214.

8. Sodhi KS, Lee EY. What all physicians should know about the potential radiation risk that computed tomography poses for paediatric patients. Acta Paediatr. 2014; 103(8): 807 - 811. 
9. Sodhi KS, Krishna S, Saxena AK, Sinha A, Khandelwal N. Lee EY. Clinical application of 'justification' and 'optimization' principle of ALARA in pediatric CT imaging: "how many children can be protected from unnecssary radiation?". Eur J Radiol. 2015; 84(9): 1752 - 1757.

10. Macdougall RD, Strauss KJ, Lee EY. Managing radiation dose from thoracic multidetector computed tomography in pediatric patients: background, current issues, and recommendations. Radiol Clin North Am. 2013; 51(4): $743-760$.

11. Qiu H, Wu J, Hong L, Luo Y, Song Q, Chen D. Clinical and epidemiological features of 36 children with coronavirus disease 2019 (COVID-19) in Zhejiang, China: an observational cohort study. Lancet Infect Dis. 2020; 20(6): 689 - 696.

12. Terpos E, Ntanasis-Stathopoulos I, Elalamy I, et al. Hematological findings and complications of COVID-19. Am J Hematol. 2020; 95(7): 834 - 847.

13. Fan BE, Chong VCL, Chan SSW, Lim GH, Lim KGE, Tan GB, et al. Hematologic parameters in patients with COVID-19 infection. Am J Hemagol. 2020;95(6): E131-E134.

14. Centers for Disease Control and Prevention. Coronavirus disease 2019 (COVID-19). Overview of testing for SARS-CoV-2: 2021. https://www.cdc.gov/coronavirus/2019-ncov/hcp/testingoverview.html. Accessed February 28, 2021.

15. Hansell DM, Bankier AA, MacMahon H, McLoud TC, Muller NL, Remy J. Fleischner Society: glossary of terms for thoracic imaging. Radiology. 2008; 245(3): 697 - 722.

16. Kundel HL, Polansky M. Measurement of observer agreement. Radiology. 2003; 228(2): 303 - 308.

17. Landis JR, Koch GG. The measurment of observer agreement for categorical data. Biometrics. 1977; 33910: 159 - 174.

18. IBM Corp. Released 2013. IBM SPSS Statistics for Windows, Version 22.0. Armonk, NY: IBM Corp.

19. Rousan LA, Elobeid E, Karrar M, Khader Y. Chest x-ray findings and temporal lung changes in patients with COVID-19 pneumonia. BMC Pulmonary Medicine. 2020; 20(1):1-9.

20. Zhou F, Yu T, Du R, Fan G, Liu Y, Liu Z, et al. Clinical course and risk factors for mortality of adult inpatients with COVID-19 in Wuhan, China: a retrospective cohort study. The lancet. 2020; 28; 395(10229): 1054 - 1062.

21. Chang T-H, Wu J-L, Chang L-Y. Clinical characteristics and diagnostic challenges of pediatric COVID19: A systematic review and meta-analysis. Journal of the Formosan Medical Association. 2020;119(5):982-9.

22. Rubin GD, Ryerson CJ, Haramati LB, Sverzellati N, Kanne JP, Raoof S, et al. The role of chest imaging in patient management during the COVID-19 pandemic: a multinational consensus statement from the Fleischner Society. Chest. 2020;158(1):106-16.

23. American College of R. ACR recommendations for the use of chest radiography and computed tomography (CT) for suspected COVID-19 infection. ACR website Advocacy-and Economics/ACRPosition-Statements/Recommendations-for-Chest-Radiography-and-CTfor-Suspected-COVID19Infection Updated March 22. 2020. Assessed: February 28, 2021. 


\section{Tables}

Table 1

\begin{tabular}{|ll|}
\hline \multicolumn{2}{|l|}{ Chest Radiographic Findings in 187 Pediatric Patients with COVID-19 Pneumonia } \\
\hline Chest Radiographic Findings & Number (Percentage) of Studies \\
\hline Normal CXR (Group 1) & $103(55)$ \\
\hline Abnormal CXR (Group 2) & $84(45 \%)$ \\
\hline Types of Abnormal CXR Findings \\
\hline GGO & $75(89 \%)$ \\
\hline GGO and Consolidation & $6(7 \%)$ \\
\hline Peribronchial Thickening & $1(1 \%)$ \\
\hline Consolidation & $1(1 \%)$ \\
\hline Peribronchial Thickening, GGO and Consolidation & $1(1 \%)$ \\
\hline
\end{tabular}

$\mathrm{CXR}=$ Chest Radiography

GGO = Ground-glass opacity

Table 2 
Comparison of Hematological Laboratory Parameters in Group 1 (with Normal CXR) Versus Group 2 (with Abnormal CXR)

Hematological Laboratory Parameters

Group $1 \quad$ Group 2

P Value

\begin{tabular}{|llll|} 
& Mean \pm SD & Mean \pm SD & \\
\hline LDH $(60-100 \mathrm{U} / \mathrm{L})$ & $200.6 \pm 53.4$ & $258.9 \pm 80.8$ & 0.001 \\
\hline C-reactive protein $(0.3$ to $1.0 \mathrm{mg} / \mathrm{dL})$ & $2.8 \pm 5.0$ & $5.7 \pm 12.3$ & 0.05 \\
\hline Serum Ferritin $(15-200 \mathrm{mg} / \mathrm{L})$ & $67.3 \pm 93.8$ & $93.2 \pm 172.0$ & 0.25 \\
\hline AST $(0-35 \mathrm{U} / \mathrm{L})$ & $24.8 \pm 15.2$ & $27.8 \pm 12.0$ & 0.22 \\
\hline ALT $(0-35 \mathrm{U} / \mathrm{L})$ & $20.2 \pm 14.0$ & $20.7 \pm 17.2$ & 0.86 \\
\hline Platelet count $(150-350 \times 109 / \mathrm{L})$ & $288.7 \pm 80.2$ & $304.2 \pm 112.3$ & 0.3 \\
\hline Lymphocytes $(1.1-3.2 \times 100$ cells per $\mathrm{L})$ & $3.0 \pm 2.4$ & $3.5 \pm 1.9$ & 0.17 \\
\hline WBC $(4-10 \times 10$ cells per $\mathrm{L})$ & $5.3 \pm 2.0$ & $5.4 \pm 2.6$ & 0.87 \\
\hline
\end{tabular}

$\mathrm{CXR}=$ Chest radiography

SD $=$ Standard deviation

$\mathrm{LDH}=$ Lactate dehydrogenase

AST $=$ Asparate aminotransferase

$\mathrm{ALT}=$ Alanine aminotransferase

WBC $=$ White blood cell

\section{Figures}




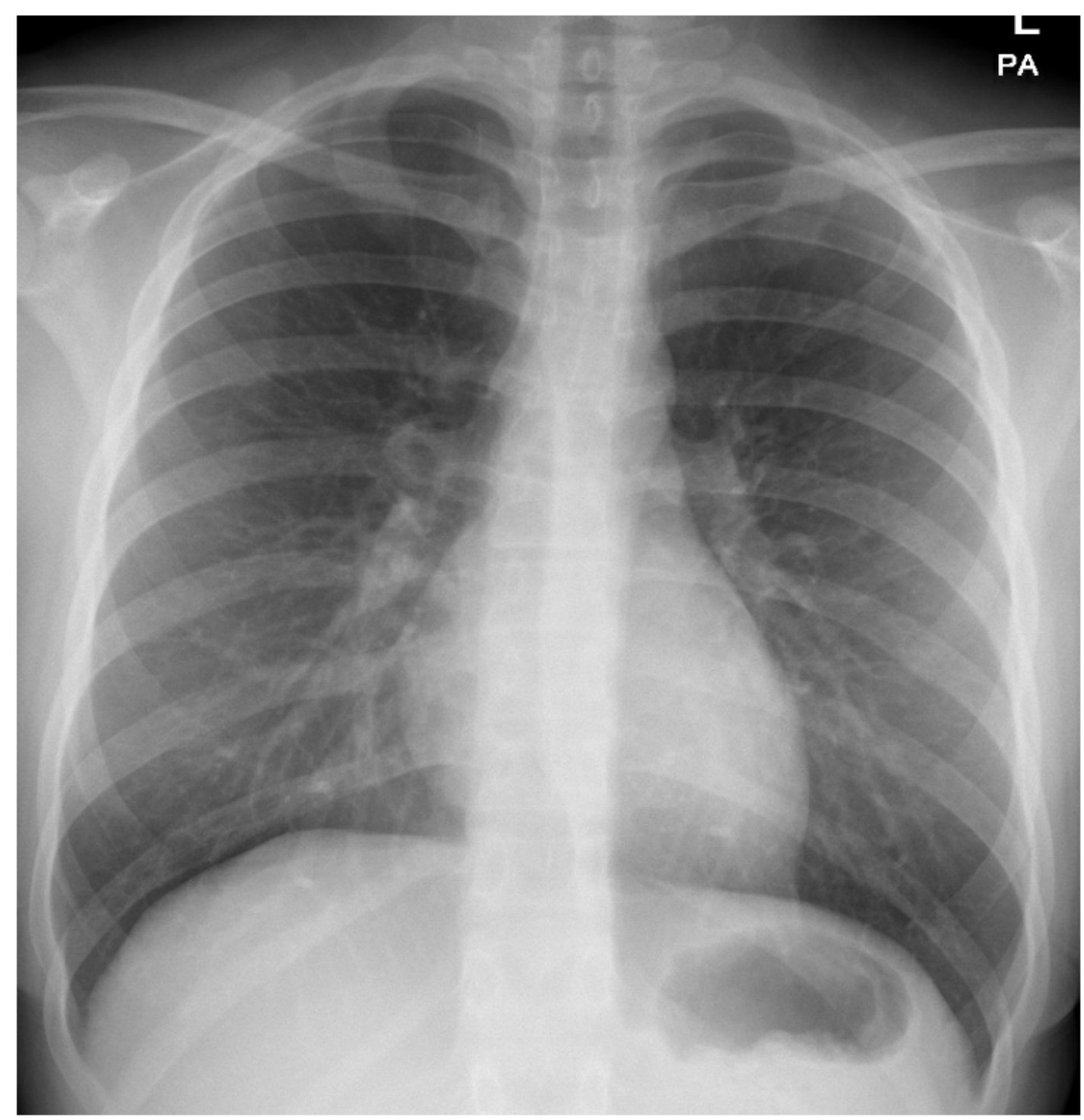

Figure 1

A 17-year-old girl with COVID-19 pneumonia based on positive RT-PCR test who presented with cough and rhinorrhea for 2 days. Frontal chest radiograph shows no radiographic abnormality. 


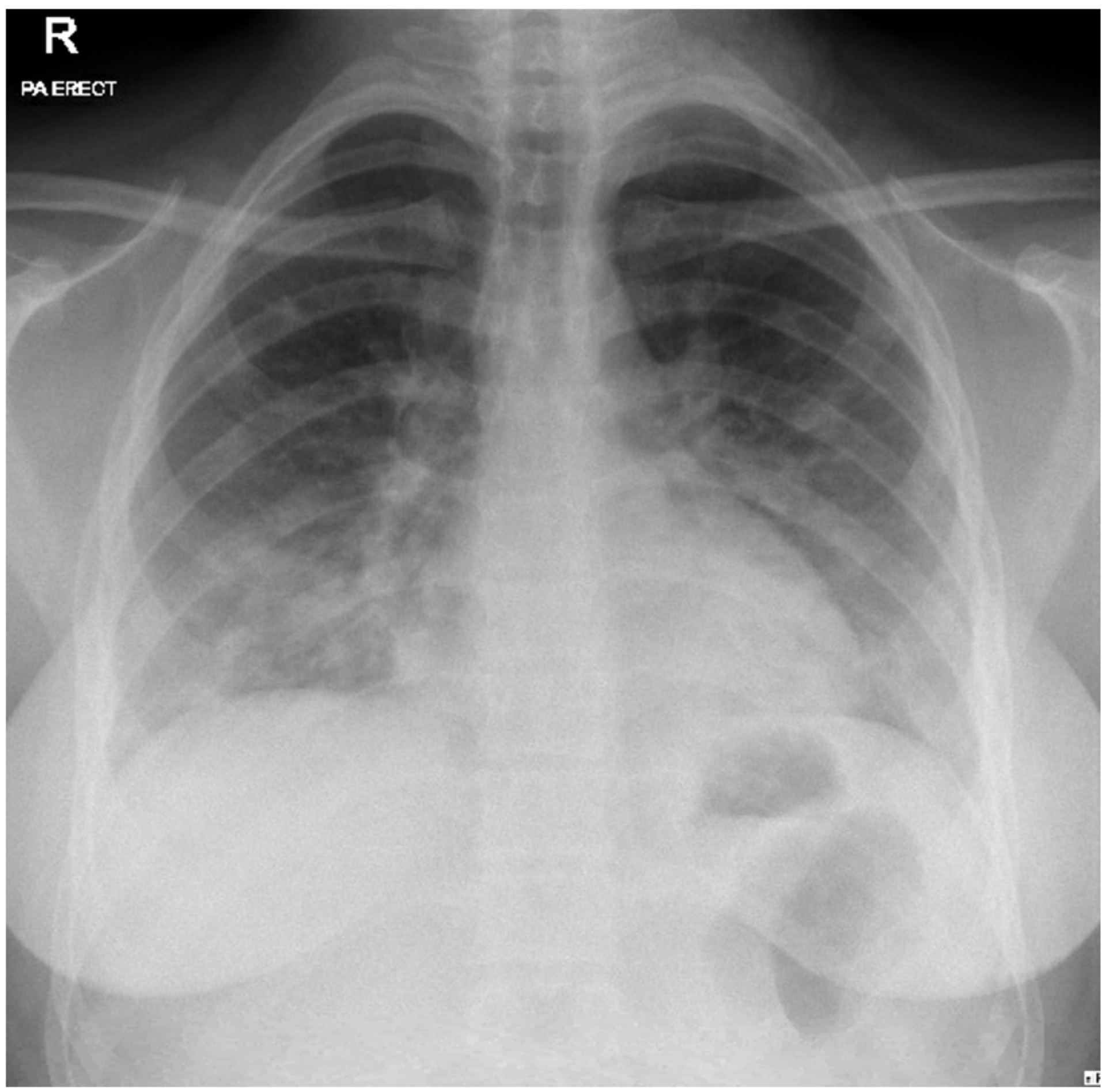

\section{Figure 2}

A 16-year-old girl with COVID-19 pneumonia based on positive RT-PCR test who presented with fever and cough for 3 days. Frontal chest radiograph shows bilateral multifocal ground-glass opacities and consolidations. The patient's hematological laboratory parameters demonstrate elevated lactate dehydrogenase level. 